\title{
Trasplante renal y rechazo vascular. Papel de enfermería en el tratamiento con plasmaféresis
}

Díaz J urado, Maribel - Díaz B arnet, Ester - Salillas Adot, Esther - E scofet Gómez, Rosa - J ulve I báñez, Maricel

Servicio de Nefrología - Hospital Universitari de Bellvitge. L'Hospitalet (Barcelona)

\section{Resumen}

La plasmaféresis (PF) es una técnica que se aplica en el servicio de trasplante renal (TR) de nuestro Hospital desde el año 1999, como tratamiento en el TR que presenta rechazo vascular. E ste rechazo puede ser de dos tipos: mediado por anticuerpos o mediado por linfocitos T. EI tratamiento con PF consigue eliminar los anticuerpos en sangre del paciente, y por tanto puede ser efectiva en el caso de que el rechazo vascular sea mediado por anticuerpos.

La técnica es Ilevada a cabo por la enfermera de la unidad de trasplante. E sta debe conocer la técnica, los parámetros, sus indicaciones y las posibles complicaciones que puedan presentarse.

EI objetivo principal de nuestro estudio es ver la efectividad del tratamiento con PF y los cuidados de enfermería que se le proporcionan.

Se realizó un estudio retrospectivo, observacional y descriptivo en una muestra de 15 pacientes. Los datos estudiados se han clasificado según el perfil

\footnotetext{
M aribel Díaz J urado

Servicio de Diálisis y Trasplante Renal

Hospital Universitario de Bellvitge

$\mathrm{C} / \mathrm{F}$ eixa Llarga $\mathrm{s} / \mathrm{n}$

Hospitalet de Llobregat. (Barcelona)

collma@wanadoo.es
}

del paciente, parámetros de la PF, evolución del $T R$, cuidados de enfermería y controles analíticos.

Los resultados obtenidos demuestran que el tratamiento con PF es eficaz en el rechazo vascular mediado por anticuerpos ofreciéndose además cuidados integrales al paciente trasplantado, ya que la enfermera de la unidad de TR es la responsable de efectuar la técnica.

\section{Palabras clave:}

TRASPLANTE RENAL

RECHAZO VASCULAR.

PLASMAFÉ RESIS

CUIDADO INTE GRAL

Kidney transplant and vascular rejection. Role of nursing in treatment with plasmapheresis

\section{Abstract}

Plasmapheresis (PPH) is a technique that has been applied in the kidney transplant service (KT) of our Hospital since 1999, as a treatment in KT that presents vascular rejection. This rejection can be of two types: caused by antibodies or caused by T lymphocytes. Treatment with P PH manages to eliminate the antibodies in the patient's blood, and can therefore be effective when the vascular rejection is caused by antibodies.

The technique is carried out by the nurse of the transplant unit. The nurse must be familiar with 
the technique, the parameters, its indications and the possible complications that may arise.

The main purpose of our study is to see the effectiveness of treatment with PPH and the nursing care provided.

A retrospective, observational and descriptive study was carried out of a sample of 15 patients. The data studied were classified according to the patient profile, PPH parameters, evolution of the $K T$, nursing care and analytical controls.

The results obtained show that treatment with PPH is efficacious in vascular rejection caused by antibodies, also offering integral care to the transplant patient, since the nurse of the KT unit is responsible for carrying out the technique.

\section{KEY WORDS: \\ KIDNEY TRANSPLANT \\ VASCULAR REJECTION. \\ PLASMAPHERESIS \\ INTE GRAL CARE}

\section{Introducción}

La plasmaféresis (PF) es una técnica que consiste en separar el plasma y sus componentes de las céIulas hemáticas, a través de una membrana de alta permeabilidad, sustituyéndolos por plasma fresco 0 sustitutos del plasma como la seroalbúmina. E s necesario disponer de un circuito hemático extracorpóreo y de un sistema de recogida del plasma filtrado y de reperfusión de la solución de sustitución.

Los cuidados de enfermería durante el tratamiento serán los propios de una técnica de hemodiálisis convencional. Como cuidados específicos están la prevención y detección de las posibles reacciones anafilácticas debido a los componentes que se perfunden, así como una correcta anticoagulación del circuito ya que aunque la duración de la PF es más corta, se trabajan con flujos de sangre que oscilan entre los 125 a $150 \mathrm{ml} / \mathrm{min}$.
La PF está indicada en enfermedades de origen inmunológico, en intoxicaciones de origen exógeno y endógeno y en el rechazo del trasplante renal, reduciendo el número de anticuerpos, entre otras.

La plasmaféresis (PF) se aplica en el servicio de Trasplante Renal (TR) de nuestro hospital desde el año 1999 como tratamiento en el trasplante renal que presenta rechazo vascular. E ste tipo de rechazo aparece precozmente (normalmente durante la primera semana), es muy severo y afecta a la supervivencia del injerto. La técnica es practicada por el personal de enfermería de la unidad de trasplante renal y forma parte de los cuidados que recibe el paciente trasplantado.

Durante el post-trasplante, ante la sospecha de rechazo, se realiza una biopsia renal (BR). En caso afirmativo, los datos obtenidos indican el grado de lesión producida en el órgano trasplantado, lesión que se clasifica según la E scala de B anff. Otro dato que aporta es la presencia de $\mathbf{c 4 d}$, la proteína que permite la unión del antígeno al anticuerpo.

Además de la biopsia, se realiza un cross-match posttrasplante, observándose que en algunos casos da resultado positivo, y por tanto hay presencia de anticuerpos frente al órgano trasplantado. La presencia de $c 4 d$, un cross-match positivo post trasplante, 0 ambos datos, indican un rechazo vascular mediado por anticuerpos, debido a la activación de linfocitos B. En el caso de que no haya confirmación de cross-match positivo $\mathrm{y} / 0$ el $\mathrm{c} 4 \mathrm{~d}$ sea negativo, se habla de rechazo vascular mediado por linfocitos $T$. Se concluye, por tanto, que hay dos tipos de rechazo vascular: el mediado por anticuerpos y el mediado por linfocitos T.

El tratamiento con plasmaféresis consigue eliminar los anticuerpos circulantes en el torrente sanguíneo del enfermo trasplantado. Para conseguir un buen intercambio plasmático es preciso disponer de un buen acceso vascular y de una correcta anticoagulación del circuito.

La enfermera responsable de llevar a cabo las sesiones de plasmaféresis debe conocer la técnica, los parámetros y sus indicaciones, así como las posibles complicaciones derivadas de ésta. 


\section{Objetivo principal}

Conocer la efectividad del tratamiento con plasmaféresis y de los cuidados de enfermería en los trasplantados renales que presentaban rechazo vascular en nuestro Hospital.

\section{Objetivos secundarios}

1. Saber la importancia de evitar complicaciones en el tratamiento con plasmaféresis, como técnica extracorpórea, en los pacientes trasplantados con rechazo vascular.

2. Valorar correctamente los parámetros referidos a la heparinización y a los accesos vasculares.

3. Adquirir conocimientos sobre el tratamiento aplicado a los trasplantados renales que presentaban rechazo vascular.

\section{Material y método}

Revisión bibliográfica referida al rechazo vascular y su tratamiento con plasmaféresis.

Revisión de las historias clínicas y de las graficas de enfermería y de plasmaféresis de los pacientes trasplantados con rechazo vascular que se realizaron PF. La muestra estudiada fue de 15 pacientes en el periodo comprendido entre 1999 y 2005.

E I diseño del estudio es retrospectivo, observacional y descriptivo. Los datos estudiados se han clasificado según: perfil del paciente, parámetros de la $P F$, evolución del $T R$, cuidados de enfermería y controles analíticos. E n el perfil del paciente, se buscó el PRA (tasa de anticuerpos citotóxicos) pre-trasplante y el PRA histórico. Se considera que un PRA superior ó igual a 50 es un paciente hipersensibilizado. Como controles analíticos se buscó el valor de la hemoglobina y el hematocrito al iniciar las sesiones de PF y al finalizar.

E $\mathrm{n}$ cada sesión se intercambió un volumen plasmático: peso $\times 5 \mathrm{ml}$.

Llamamos bed side (al pie de la cama) a la realización de la PF en la unidad de trasplante y por el mismo personal de enfermería responsable de los pacientes trasplantados (figura 1).

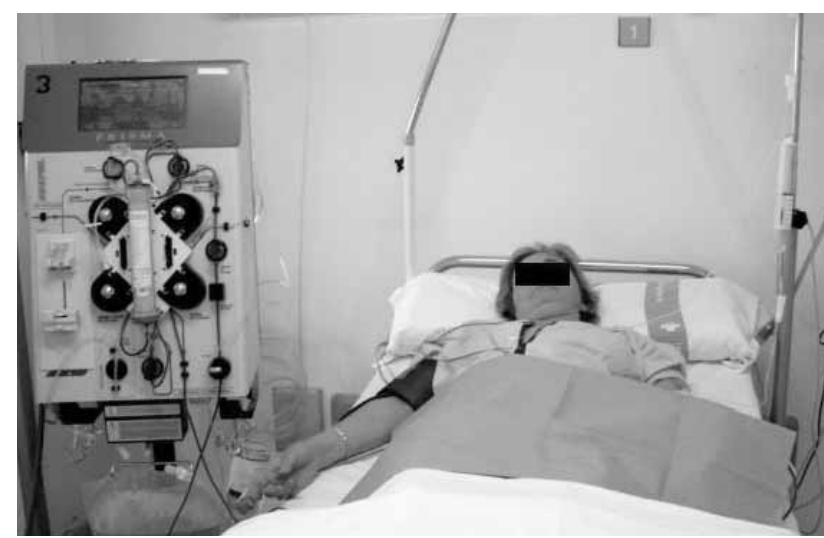

Figura 1. Realización de la plasmaféresis

\section{Resultados}

\section{Perfil del paciente}

L a media de edad de los pacientes estudiados era de $49,6 \pm 10,9$ años, de los cuales el $53,3 \%$ eran mujeres y el $46,6 \%$, hombres. Según el número de trasplantes: $\mathbf{5 0 \%}$ era un segundo trasplante, el $\mathbf{1 7 \%}$, un tercer trasplante y un $33 \%$, el primero (figura 2 ).

E ncontramos en el momento del trasplante un 13\% de pacientes hipersensibilizados frente a un $87 \%$ que no lo estaban. Según el PRA histórico, un $47 \%$ de los pacientes estuvieron alguna vez hipersensibilizados, mientras que un $53 \%$ no.

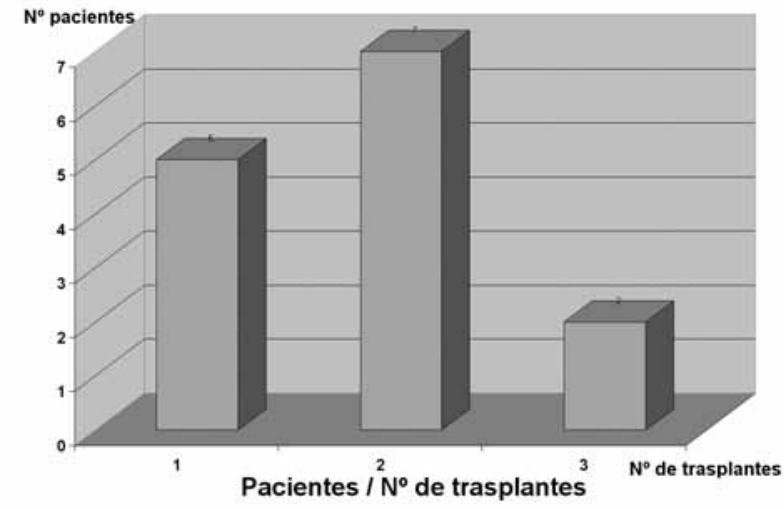

Figura 2. Trasplantes por paciente

\section{Parámetros de la plasmaféresis}

L a media del número de sesiones de PF fue de 4,3 \pm 1,2 sesiones, y el promedio de tiempo de tratamiento, de 11,26 $\pm 3,5$ horas (figuras 3 y 4). La media de horas por sesión fue de 2,31 $\pm 0,4$ horas. Se utilizaron flujos de una media de $142 \pm 17,7 \mathrm{ml} /$ minuto. 


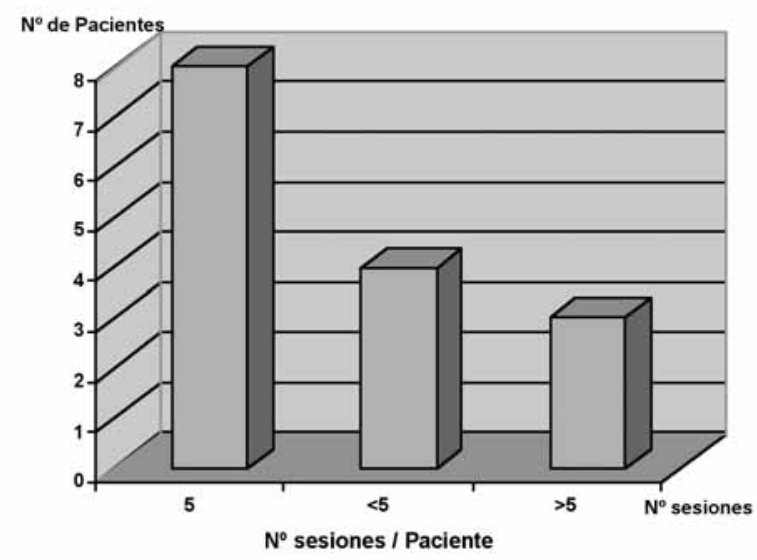

Figura 3. Sesiones de plasmaféresis por paciente

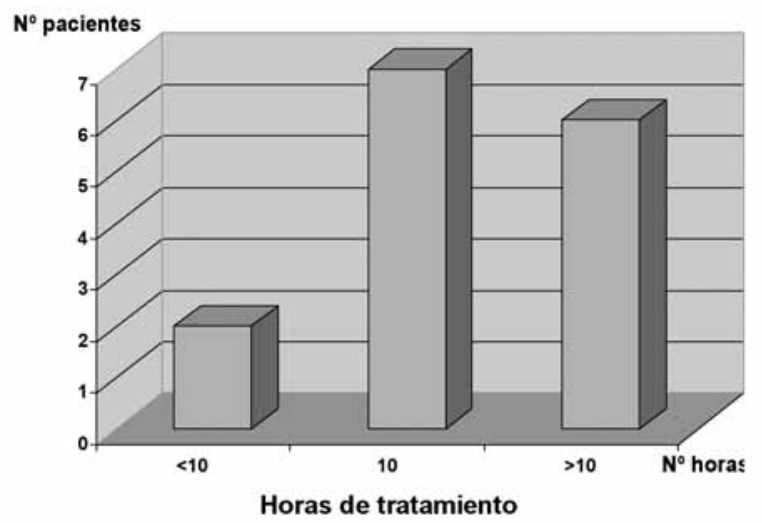

Figura 4. Horas de tratamiento con plasmaféresis

Un $\mathbf{8 0 \%}$ de pacientes llevaron heparina sódica durante todas las sesiones de PF, un $7 \%$ heparina cálcica y en un $13 \%$ no se utilizó ningún tipo de heparina (figura 5). De los pacientes que llevaron heparina sódica, la media de dosis inicial fue $15,1 \pm 7,9$ $\mathrm{mg}$ y la de mantenimiento $3,9 \pm 1,9 \mathrm{mg}$

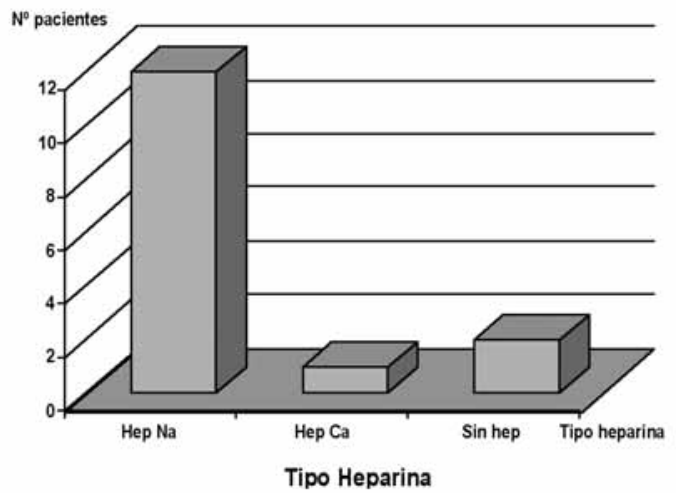

Figura 5. Tipos de heparina utilizada durante la plasmaféresis
La media de albúmina intercambiada por sesión fue de $2864 \pm 669,2 \mathrm{ml}$ y la de plasma fresco, de 651,2 $\pm 192,3 \mathrm{ml}$. No a todos los pacientes se les administró plasma fresco, sólo al $67 \%$, porque presentaban problemas de coagulación.

\section{E volución del Trasplante}

La supervivencia del injerto fue de un $67 \%$, Un $60 \%$ precisó tratamiento renal sustitutivo durante el posttrasplante. La media de días transcurridos de:

1) TR a BR: $10,1 \pm 7,36$ días.

2) BR a PF: $1,85 \pm 4,1$ días.

3) TR a PF: $11,8 \pm 9,1$ días.

Por tanto en un $\mathbf{5 0 \%}$ se realizó la PF al día siguiente de la BR, un $7 \%$ el mismo día y un $43 \%$ al cabo de dos 0 más días (figura 6 ). Del resultado de la BR en un $47 \%$ se encontró la presencia de c $4 d$ positivo.

En un $\mathbf{4 0 \%}$ de los pacientes trasplantados se pudo realizar un cross-match post-trasplante, con resultado positivo en un $33 \%$ de los casos.

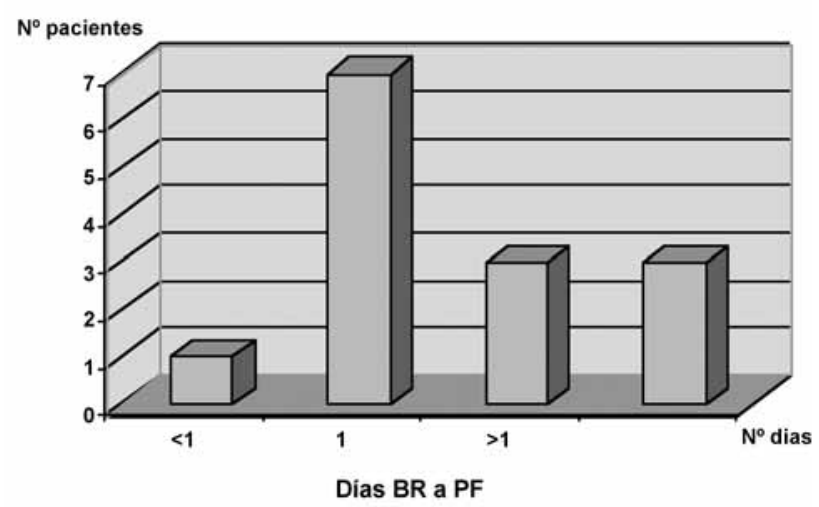

Figura 6. Tiempo transcurrido entre la biopsia renal y la plasmaféresis

Cuidados de enfermería respecto al riesgo de sangrado/coagulación del circuito y el acceso vascular Se realizó la PF en la unidad de TR en un $73 \%$ (bed side). Ningún paciente sangró por la herida quirúrgica como consecuencia de la administración de heparina para la sesión de PF en el post operatorio inmediato. Sin embargo, tres pacientes presentaron sangrado a medio o largo plazo relacionado con la 
administración de heparina, lo que se manifestó por: hematoma en injerto post BR, hematoma en herida quirúrgica e hipermenorrea. En este último caso se precisó transfusión de dos concentrados de hematíes. Los dos primeros no requirieron ningún tratamiento.

El $27 \%$ de los pacientes todavía tenían redón cuando se les hizo el tratamiento. Ninguno de ellos presentó un aumento de la producción del redón.

De los 74 circuitos realizados, el $11 \%$ se coagularon, frente a un $89 \%$ que no presentaron problemas de este tipo. El $87 \%$ de los circuitos coagulados fue debido a complicaciones del acceso vascular. Un $20 \%$ de los pacientes presentaron, en alguna de las sesiones 0 en todas ellas, problemas con el acceso vascular. Dichos problemas fueron: un catéter femoral que no daba buen flujo y una extravasación del tramo venoso en una FAVI. EI catéter femoral se colocó en un paciente que estaba en programa de diálisis peritoneal y no tenia buenos accesos vasculares.

\section{Controles analíticos}

El promedio del hematocrito al inicio fue del 30,22 $\pm 6,3 \%$ con una hemoglobina de $10 \pm 2,2 \mathrm{mg} / \mathrm{dl}$. Al terminar la última sesión de PF, fue del 27,26 \pm 5,9 $\%$ con una hemoglobina de $8,84 \pm 1,8 \mathrm{mg} / \mathrm{dll}$. Se observó una disminución general de 2,95\% del hematocrito y un $1,16 \mathrm{mg} / \mathrm{dl}$ de la hemoglobina.

\section{E fectividad de la plasmaféresis}

E n nuestra muestra, un $71 \%$ de los pacientes con rechazo vascular mediado por anticuerpos que se trataron con PF consiguieron la supervivencia del injerto.

\section{Discusión}

E I personal especializado de la unidad de trasplante realizó la PF bed side a todos los pacientes que presentaron rechazo vascular durante la primera semana del trasplante. De esta manera la enfermera que sigue la evolución de la herida quirúrgica, el control de los redones y los cuidados propios de una BR entre otros, es también la responsable de efectuar la sesión de PF, ofreciendo un cuidado global. A los pacientes que no eran recién trasplantados, 0 sea que presentaron rechazo vascular pasados al menos 25 días post TR, no se les realizó la PF bed side.

P ara determinar las consecuencias de la administración de heparina en el tratamiento en pacientes recién trasplantados y biopsiados, se observó el posible sangrado por la herida quirúrgica, el redón, los sangrados tardíos (algunos de ellos detectados por pruebas diagnósticas) y la caída del hematocrito y hemoglobina. EI hecho de que la herida quirúrgica no sangrara de manera visible en ningún caso, se debe probablemente a que las dosis de heparina estaban muy ajustadas a cada paciente y se utilizó mayoritariamente heparina de corta duración. E n nuestra unidad se retira el redón por protocolo al cuarto día, siempre que éste ya no sea productivo. E $n$ la mayoría de los casos, la PF se realizó al 11 día. Por tanto no pudimos valorar un sangrado por el redón, ya que a la mayor parte de ellos se les había retirado el drenaje.

A unque hay una disminución del hematocrito y la hemoglobina entre el primer y el último día de la PF, no se puede ver una relación directa entre estos factores, ya que todos los pacientes trasplantados presentan una anemia debida a la cirugía y a la propia insuficiencia renal.

Con la administración de heparina conseguimos que el $89 \%$ de los circuitos no se coagularan. Las causas de coagulación fueron debidas principalmente al mal funcionamiento del acceso vascular.

A tres de los siete pacientes con presencia de $c 4 d$ positivo se les realizó un cross-match post-trasplante para confirmar la presencia de rechazo humoral. E sto es debido a que solo recientemente se guarda muestra de sangre del donante. Por otra parte, dos de estos tres pacientes dieron cross-match positivo. De esto se deduce que no es necesaria la presencia de $\mathrm{c} 4 \mathrm{~d}$ positivo y cross match positivo para definir un rechazo vascular como humoral, sino que la presencia de uno de ellos ya es concluyente.

\section{Conclusiones}

Al ser la enfermera de la unidad de trasplante renal la responsable de realizar la PF, se consiguen ofrecer 
unos cuidados integrales al paciente trasplantado con rechazo vascular.

E $n$ el tratamiento con PF en el trasplantado renal con rechazo vascular mediado por anticuerpos, está justificada la administración de heparina dada la importancia de mantener un circuito anticoagulado para conseguir un buen intercambio plasmático.

Cuando se tienen buenos accesos vasculares y se manipulan correctamente, se consiguen realizar con éxito las sesiones de plasmaféresis.

La PF como tratamiento en el rechazo vascular mediado por anticuerpos es eficaz y logra una elevada supervivencia del injerto.

\section{Bibliografia}

1. Botella García J. Manual de Nefrología Clínica. Barcelona: Masson; 2003.
2. B urns Grove. Investigación en E nfermería. 3a E dición. Madrid: E Isevier; 2004.

3. Anaya F. Aféresis Terapéutica. Madrid: Capitel; 2005.

4. González M olina M, Alonso Ortiz A. B iología de la Inmunosupresión del Trasplante de Órganos. Barcelona: Novartis Farmacéutica S A; 2005.

5. SE DE N. M anual de Protocolos y Procedimientos de E nfermería N efrológica. M adrid: Grupo E nthos S L; 2001.

6. Andreu L, Force E. La E nfermería y el Trasplante de Órganos. Madrid: Panamericana; 2005.

7. Andres J , Fortuny C. Cuidados de E nfermería en la Insuficiencia Renal. Barcelona: Gallery Heathcom; 1998.

8. Andreu Periz L, Force Sanmartín E . 500 Cuestiones que plantea el cuidado del enfermo renal. Barcelona: Masson S.A.; 2001. 